\title{
OAIAA \\ AIAA 2004-0317 \\ Numerical Simulation of 3-D Supersonic Viscous Flow in an Experimental MHD Channel
}

Hiromasa Kato, John C. Tannehill, Sumeet Gupta, lowa State University, Ames, IA 50011

and

Unmeel B. Mehta

NASA Ames Research Center, Moffett Field, CA 94035

\section{2nd AIAA Aerospace Sciences Meeting and Exhibit 5-8 January 2004 / Reno, NV}




\title{
Numerical Simulation of 3-D Supersonic Viscous Flow in an Experimental MHD Channel
}

\author{
Hiromasa Kato*, John C. Tannehill ${ }^{\dagger}$, Sumeet Gupta* \\ Iowa State University, Ames, IA 50011 \\ and \\ Unmeel B. Mehta ${ }^{\ddagger}$ \\ NASA Ames Research Center, Moffett Field, CA 94035
}

\begin{abstract}
The 3-D supersonic viscous flow in an experimental MHD channel has been numerically simulated. The experimental $\mathrm{MHD}$ channel is currently in operation at NASA Ames Research Center. The channel contains a nozzle section, a center section, and an accelerator section where magnetic and electric fields can be imposed on the flow. In recent tests, velocity increases of up to $40 \%$ have been achieved in the accelerator section. The flow in the channel is numerically computed using a new 3-D parabolized NavierStokes (PNS) algorithm that has been developed to efficiently compute MHD flows in the low magnetic Reynolds number regime. The MHD effects are modeled by introducing source terms into the PNS equations which can then be solved in a very efficient manner. To account for upstream (elliptic) effects, the flowfield can be computed using multiple streamwise sweeps with an iterated PNS algorithm. The new algorithm has been used to compute two test cases that match the experimental conditions. In both cases, magnetic and electric fields are applied to the flow. The computed results are in good agreement with the available experimental data.
\end{abstract}

\section{Introduction}

Magnetohyrodynamics (MHD) can be utilized to improve performance and extend the operational range of many systems. Potential applications include hypersonic cruise, advanced Earth-to-orbit propulsion, chemical and nuclear space propulsion, regenerative

\footnotetext{
- Graduate Research Assistant, Student Member AIAA

†Manager, Computational Fluid Dyzamics Center, and Professor, Dept. of Aerospace Engineering. Fellow AIAA

$\$$ Division Scientist, Associate Fellow, ALAA

Copyright (C2004 by the American Institute of Aeronautics and Astronautics, Inc,, all rights reserved.
}

aerobraking, onboard flow control systems, test facilities, launch assist, and power generation. One of the critical technologies associated with these applications is MHD acceleration. In order to study MHD acceleration, an experimental MHD channel has been built at NASA Ames Research Center by D. W. Bogdanoff, C. Park, and U. B. Mehta [1,2]. The channel is about a half meter long and contains a nozzle section, a center section, and an accelerator section. The channel has a uniform width of $2.03 \mathrm{~cm}$. Magnetic and electric fields can be imposed upon the flow in the accelerator section. A cross section of the MHD channel is shown in Fig. 1.

In the present study, the flow in the experimental MHD channel is numerically simulated. Flowfields involving MHD effects have typically been computed [3-15] by solving the complete NavierStokes (N-S) equations for fluid flow in conjunction with Maxwell's equations of electromagnetodynamics. When chemistry and turbulence effects are also included, the computational effort required to solve the resulting coupled system of partial differential equations is extremely formidable. One possible remedy to this problem is to use the parabolized NavierStokes (PNS) equations in place of the N-S equations. The PNS equations can be used to compute three-dimensional, supersonic viscous flowfields in a very efficient manner [16]. This efficiency is achieved because the equations can be solved using a spacemarching technique as opposed to the time-marching technique that is normally employed for the complete N-S equations.

Recently, the present authors have developed PNS codes to solve 2-D supersonic MHD flowfields in both the high and low magnetic Reynolds number regimes $[17,18]$ : The magnetic Reynolds number is defined as $R e_{m}=\sigma_{e} \mu_{e} V_{\infty} L$ where $\sigma_{e}$ is the electrical conductivity, $\mu_{e}$ is the magnetic permeability, $V_{\infty}$ is the freestream velocity, and $L$ is the reference length. 
The new MHD PNS codes are based on NASA's upwind PNS (UPS) code which was originally developed by Lawrence et al. [19]. The UPS code solves the PNS equations using a fully conservative, finitevolume approach in a general nonorthogonal coordinate system. The UPS code has previously been extended to permit the computation of flowfields with strong upstream influences. In regions where strong upstream influences are present, the governing equations are solved using multiple sweeps (i.e. iterations). As a result of this approach; a complete flowfield can be computed more efficiently (in terms of computer time and storage) than with a standard N$S$ solver which marches the entire solution in time. Three iterative PNS algorithms called IPNS, TIPNS, and FBIPNS have been developed and are described in Refs. [20-22].

For many aerospace applications, including the present experimental MHD channel, the electrical conductivity of the fluid is low and hence the magnetic Reynolds number is small. In these cases, it makes sense to use the low magnetic Reynolds number assumption and reduce the complexity of the governing equations. The MHD effects are modeled with the introduction of source terms into the fluid flow equations, as was done in the present low magnetic Reynolds number PNS code [18]. This code has been extended to three-dimensions in the present study and is used for the numerical simulations of the flow in the experimental MHD channel.

\section{Governing Equations}

The governing equations for a viscous MHD flow with a small magnetic Reynolds number are given by [14]:

Continuity equation

$$
\frac{\partial \rho}{\partial t}+\nabla \cdot(\rho \mathbf{V})=0
$$

Momentum equation

$$
\frac{\partial(\rho \mathbf{V})}{\partial t}+\nabla \cdot[\rho \mathbf{V V}+p \overline{\overline{\mathbf{I}}}]=\nabla \cdot \overline{\bar{\tau}}+\mathbf{J} \times \mathbf{B}
$$

Energy equation

$$
\frac{\partial\left(\rho e_{t}\right)}{\partial t}+\nabla \cdot\left[\left(\rho e_{t}+p\right) \mathbf{V}\right]=\nabla \cdot(\mathbf{V} \cdot \overline{\bar{\tau}})-\nabla \cdot \mathbf{U}+\mathbf{E} \cdot \mathbf{J}
$$

Ohm's law

$$
\mathbf{J}=\sigma_{e}(\mathbf{E}+\mathbf{V} \times \mathbf{B})
$$

where $\mathbf{V}$ is the velocity vector, $B$ is the magnetic field vector, $\mathbf{E}$ is the electric field vector, and $\mathbf{J}$ is the conduction current density.
The governing equations are nondimensionalized using the following reference variables:

$$
\begin{gathered}
x^{*}, y^{*}, z^{*}=\frac{x, y, z}{L}, \quad u^{*}, v^{*}, w^{*}=\frac{u, v, w}{U_{\infty}}, \quad t^{*}=\frac{U_{\infty} t}{L} \\
\rho^{*}=\frac{\rho}{\rho_{\infty}}, \quad T^{*}=\frac{T}{T_{\infty}}, \quad p^{*}=\frac{p}{\rho_{\infty} U_{\infty}^{2}} \\
e_{t}^{*}=\frac{e_{t}}{U_{\infty}^{2}}, \quad \bar{\tau}^{*}=\frac{\bar{\tau} L}{\mu_{\infty} U_{\infty}}, \quad \mu^{*}=\frac{\mu}{\mu_{\infty}} \quad(5) \\
B_{x}^{*}, B_{y}^{*}, B_{z}^{*}=\frac{B_{x}, B_{y}, B_{z}}{U_{\infty} \sqrt{\mu_{e} \rho_{\infty}}}, \quad E_{x}^{*}, E_{y}^{*}, E_{z}^{*}=\frac{E_{x}, E_{y}, E_{z}}{U_{\infty}^{2} \sqrt{\mu_{e} \rho_{\infty}}} \\
\mu_{e}^{*}=\frac{\mu_{e}}{\mu_{e \infty}}=1, \quad \sigma_{e}^{*}=\frac{\sigma_{e}}{\sigma_{e \infty}}
\end{gathered}
$$

where the superscript * refers to the nondimensional quantities. For convenience, the asterisks are dropped in the following equations.

The governing equations written in vector form in a 3-D Cartesian coordinate system become

$\frac{\partial \mathrm{U}}{\partial t}+\frac{\partial \mathbf{E}_{i}}{\partial x}+\frac{\partial \mathbf{F}_{i}}{\partial y}+\frac{\partial \mathbf{G}_{i}}{\partial z}=\frac{\partial \mathbf{E}_{v}}{\partial x}+\frac{\partial \mathbf{F}_{v}}{\partial y}+\frac{\partial \mathbf{G}_{v}}{\partial z}+\mathbf{S}_{\mathrm{MHD}}$

where $U$ is the vector of dependent variables, $\mathbf{E}_{i}, \mathbf{F}_{i}$ and $G_{i}$ are the inviscid flux vectors, and $E_{v}, F_{v}$ and $G_{v}$ are the viscous flux vectors. The source term

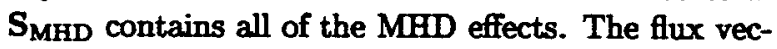
tors are given by

$$
\mathrm{U}=\left[\begin{array}{lllll}
\rho, & \rho u, & \rho v, & \rho w, & \rho e_{t}
\end{array}\right]^{T}
$$

$$
\begin{gathered}
\mathbf{E}_{i}=\left[\begin{array}{c}
\rho u \\
\rho u^{2}+p \\
\rho u v \\
\rho u w \\
\left(\rho e_{t}+p\right) u
\end{array}\right], \mathbf{F}_{i}=\left[\begin{array}{c}
\rho v \\
\rho u v \\
\rho v^{2}+p \\
\rho v w \\
\left(\rho e_{t}+p\right) v
\end{array}\right] \\
\mathbf{G}_{i}=\left[\begin{array}{c}
\rho w \\
\rho u w \\
\rho v w \\
\rho w^{2}+p \\
\left(\rho e_{t}+p\right) w
\end{array}\right]
\end{gathered}
$$

$$
\mathrm{E}_{v}=\left[\begin{array}{c}
0 \\
\tau_{x x} \\
\tau_{x y} \\
\tau_{x z} \\
u \tau_{x x}+v \tau_{x y}+w \tau_{x z}-q_{x}
\end{array}\right]
$$

$$
F_{v}=\left[\begin{array}{c}
0 \\
\tau_{y x} \\
\tau_{y y} \\
\tau_{y z} \\
u \tau_{y x}+v \tau_{y y}+w \tau_{y z}-q_{y}
\end{array}\right]
$$




$$
\begin{gathered}
\mathbf{G}_{v}=\left[\begin{array}{c}
0 \\
\tau_{z x} \\
\tau_{z y} \\
\tau_{z z} \\
u \tau_{z x}+v \tau_{z y}+w \tau_{z z}-q_{z}
\end{array}\right] \\
\mathrm{S}_{\mathrm{MHD}}=R e_{m}\left[\begin{array}{c}
0 \\
\hline B_{z}\left(E_{y}+w B_{z}-u B_{z}\right) \\
-B_{y}\left(E_{z}+u B_{y}-v B_{x}\right) \\
\hline B_{x}\left(E_{z}+u B_{y}-v B_{z}\right) \\
-B_{z}\left(E_{z}+v B_{z}-w B_{y}\right) \\
\hline B_{y}\left(E_{x}+v B_{z}-w B_{y}\right) \\
-B_{z}\left(E_{y}+w B_{x}-u B_{z}\right) \\
\hline E_{x}\left(E_{x}+v B_{z}-w B_{y}\right) \\
+E_{y}\left(E_{y}+w B_{x}-u B_{z}\right) \\
+E_{z}\left(E_{z}+u B_{y}-v B_{z}\right)
\end{array}\right]
\end{gathered}
$$

where

$$
\rho e_{t}=\frac{1}{2} \rho\left(u^{2}+v^{2}+w^{2}\right)+\frac{p}{\bar{\gamma}-1}
$$

and the nondimensional shear stresses and heat fluxes are defined in the usual manner [16].

The flow can be computed assuming either a constant $\bar{\gamma}$ or by using the simplified curve fits of Srinivasan et al. $[23,24]$ for the thermodynamic and transport properties of equilibrium air. For the latter case, $\bar{\gamma}$ in Equation (13) is determined using the curve fit $\tilde{\gamma}=\bar{\gamma}(e, \rho)$. In future calculations, the flow will be computed in chemical nonequilibrium.

The governing equations are transformed into computational space and written in a generalized coordinate system $(\xi, \eta, \zeta)$ as

$$
\frac{1}{J} \mathbf{U}_{t}+\tilde{\mathbf{E}}_{\xi}+\tilde{\mathbf{F}}_{\eta}+\tilde{\mathbf{G}}_{\zeta}=\frac{\mathbf{S}_{\mathrm{MHD}}}{J}
$$

where

$$
\begin{aligned}
\tilde{\mathbf{E}}= & \left(\frac{\xi_{x}}{J}\right)\left(\mathbf{E}_{i}-\mathbf{E}_{v}\right)+\left(\frac{\xi_{y}}{J}\right)\left(\mathbf{F}_{i}-\mathbf{F}_{v}\right) \\
& +\left(\frac{\xi_{z}}{J}\right)\left(\mathbf{G}_{i}-\mathbf{G}_{v}\right) \\
\tilde{\mathbf{F}}= & \left(\frac{\eta_{x}}{J}\right)\left(\mathbf{E}_{i}-\mathbf{E}_{v}\right)+\left(\frac{\eta_{v}}{J}\right)\left(\mathbf{F}_{i}-\mathbf{F}_{v}\right) \\
& +\left(\frac{\eta_{z}}{J}\right)\left(\mathbf{G}_{i}-\mathbf{G}_{v}\right) \\
\tilde{\mathbf{G}}= & \left(\frac{\zeta_{x}}{J}\right)\left(\mathbf{E}_{i}-\mathbf{E}_{v}\right)+\left(\frac{\zeta_{y}}{J}\right)\left(\mathbf{F}_{i}-\mathbf{F}_{v}\right) \\
& +\left(\frac{\zeta_{z}}{J}\right)\left(\mathbf{G}_{i}-\mathbf{G}_{v}\right)
\end{aligned}
$$

and $J$ is the Jacobian of the transformation.

The governing equations are parabolized by dropping the time derivative term and the streamwise direction $(\xi)$ viscous flow terms in the flux vectors. Equation (14) can then be rewritten as

$$
\tilde{\mathbf{E}}_{\xi}+\tilde{\mathbf{F}}_{\eta}+\tilde{\mathbf{G}}_{\zeta}=\frac{\mathbf{S}_{\mathrm{MHD}}}{J}
$$

where

$$
\begin{aligned}
\dot{\mathbf{E}}= & \left(\frac{\xi_{x}}{J}\right) \mathbf{E}_{i}+\left(\frac{\xi_{y}}{J}\right) \mathbf{F}_{i}+\left(\frac{\xi_{z}}{J}\right) \mathbf{G}_{i} \\
\dot{\mathbf{F}}= & \left(\frac{\eta_{x}}{J}\right)\left(\mathbf{E}_{i}-\mathbf{E}_{v}^{\prime}\right)+\left(\frac{\eta_{y}}{J}\right)\left(\mathbf{F}_{i}-\mathbf{F}_{v}^{\prime}\right) \\
& +\left(\frac{\eta_{z}}{J}\right)\left(\mathbf{G}_{i}-\mathbf{G}_{v}^{\prime}\right) \\
\tilde{\mathbf{G}}= & \left(\frac{\zeta_{x}}{J}\right)\left(\mathbf{E}_{i}-\mathbf{E}_{v}^{\prime}\right)+\left(\frac{\zeta_{y}}{J}\right)\left(\mathbf{F}_{i}-\mathbf{F}_{v}^{\prime}\right) \\
& +\left(\frac{\zeta_{z}}{J}\right)\left(\mathbf{G}_{i}-\mathbf{G}_{v}^{\prime}\right)
\end{aligned}
$$

The primes in the preceding equations indicate that the streamwise viscous flow terms have been dropped.

For turbulent flows, the two-layer Baldwin-Lomax turbulence model [25] has been modified to account for MHD effects. Only the expression for turbulent viscosity in the inner layer is changed. This modifcation for MHD flows is due to Lykoudis [26].

\section{Numerical Method}

The governing PNS equations with MHD source terms have been incorporated into NASA's upwind PNS (UPS) code [19]. These equations can be solved very efficiently using a single sweep of the flowfield for many applications. For cases where upstream (elliptic) effects are important, the flowfield can be computed using multiple streamwise sweeps with either the IPNS [20], TIPNS [21], or FBIPNS [22] algorithms. This iterative process is continued until the solution is converged.

For the iterative PNS (IPNS) method, the $\mathbf{E}$ vector is split using the Vigneron parameter $(\omega)$ [27]. This parameter does not need to be changed for the present low magnetic Reynolds number formulation. In the previous high magnetic Reynolds number code [17] it was necessary to modify the Vigneron parameter to account for MHD effects. After splitting, the $\overline{\mathbf{E}}$ vector can be written as:

$$
\tilde{\mathbf{E}}=\mathbf{E}^{*}+\mathbf{E}^{p}
$$

where

$$
\mathbf{E}^{*}=\frac{\xi_{x}}{J}\left[\begin{array}{c}
\rho u \\
\rho u^{2}+\omega p \\
\rho u v \\
\rho u w \\
\left(\rho e_{t}+p\right) u
\end{array}\right]+\frac{\xi_{y}}{J}\left[\begin{array}{c}
\rho v \\
\rho u v \\
\rho v^{2}+\omega p \\
\rho v w \\
\left(\rho e_{t}+p\right) v
\end{array}\right]
$$




$$
\begin{aligned}
&+ \frac{\xi_{z}}{J}\left[\begin{array}{c}
\rho w \\
\rho u w \\
\rho v w \\
\rho \omega^{2}+\omega p \\
\left(\rho e_{t}+p\right) w
\end{array}\right] \\
& 0 \\
& \mathbf{E}^{p}= \frac{\xi_{x}}{J}\left[\begin{array}{c}
(1-\omega) p \\
0 \\
0 \\
0
\end{array}\right]+\frac{\xi_{z}}{J}\left[\begin{array}{c}
0 \\
0 \\
(1-\omega) p \\
0 \\
0 \\
0 \\
0 \\
0 \\
(1-\omega) p \\
0
\end{array}\right] \\
&+ \frac{\xi_{z}}{J}\left[\begin{array}{c} 
\\
0
\end{array}\right]
\end{aligned}
$$

The streamwise derivative of $\bar{E}$ is then differenced using a backward difference for $\mathbf{E}^{*}$ and a forward difference for the "elliptic" portion $\left(\mathbf{E}^{p}\right)$ :

$$
\left(\frac{\partial \overline{\mathbf{E}}}{\partial \xi}\right)_{i+1}=\frac{1}{\Delta \xi}\left[\left(\mathbf{E}_{i+1}^{*}-\mathbf{E}_{i}^{*}\right)+\left(\mathbf{E}_{i+2}^{p}-\mathbf{E}_{i+1}^{p}\right)\right]
$$

where the subscript $(i+1)$ denotes the spatial index (in the $\xi$ direction) where the solution is currently being computed. The vectors $\mathbf{E}_{i+1}^{*}$ and $\mathbf{E}_{i+1}^{p}$ are then linearized in the following manner:

$$
\begin{aligned}
& \mathbf{E}_{i+1}^{*}=\mathbf{E}_{i}^{*}+\left(\frac{\partial \mathbf{E}^{*}}{\partial \mathbf{U}}\right)_{i}\left(\mathbf{U}_{i+1}-\mathbf{U}_{i}\right) \\
& \mathbf{E}_{i+1}^{p}=\mathbf{E}_{i}^{p}+\left(\frac{\partial \mathbf{E}^{p}}{\partial \mathbf{U}}\right)_{i}\left(\mathbf{U}_{i+1}-\mathbf{U}_{i}\right)
\end{aligned}
$$

The Jacobians can be represented by

$$
\begin{aligned}
& A^{*}=\frac{\partial \mathbf{E}^{*}}{\partial \mathbf{U}} \\
& A^{p}=\frac{\partial \mathbf{E}^{p}}{\partial \mathbf{U}}
\end{aligned}
$$

After substituting the above linearizations into Eq. (20), the expression for the streamwise gradient of $\tilde{\mathbf{E}}$ becomes

$$
\begin{aligned}
\left(\frac{\partial \overline{\mathrm{E}}}{\partial \xi}\right)_{i+1}= & \frac{1}{\Delta \xi}\left[\left(A_{i}^{*}-A_{i}^{p}\right)\left(\mathrm{U}_{i+1}-\mathrm{U}_{i}\right)\right. \\
& \left.+\left(\mathrm{E}_{i+2}^{p}-\mathrm{E}_{i}^{p}\right)\right]
\end{aligned}
$$

The final discretized form of the fluid flow equations with MHD source terms is obtained by substituting Eq. (23) into Eq. (16) along with the linearized expressions for the fluxes in the cross flow plane. The final expression becomes:

$$
\begin{gathered}
{\left[\frac{1}{\Delta \xi}\left(A_{i}^{*}-A_{i}^{p}\right)+\frac{\partial}{\partial \eta}\left(\frac{\partial \overline{\mathbf{F}}}{\partial \overline{\mathrm{U}}}\right)_{i}+\frac{\partial}{\partial \zeta}\left(\frac{\partial \tilde{\mathbf{G}}}{\partial \overline{\mathrm{U}}}\right)_{i}\right]^{k+1}\left(\Delta \mathrm{U}_{i}\right)^{k+1}} \\
=\text { RHS }
\end{gathered}
$$

where

$$
\begin{aligned}
&\left(\Delta \mathrm{U}_{i}\right)^{k+1}=\left(\mathrm{U}_{i+1}-\mathrm{U}_{i}\right)^{k+1} \\
& \text { RHS }=-\frac{1}{\Delta \xi}\left[\left(\mathrm{E}_{i+2}^{p}\right)^{k}-\left(\mathbf{E}_{i}^{p}\right)^{k+1}\right]-\left(\frac{\partial \tilde{\mathbf{F}}}{\partial \eta}\right)_{i}^{k+1} \\
&-\left(\frac{\partial \tilde{\mathrm{G}}}{\partial \zeta}\right)_{i}^{k+1}+\left(\frac{\mathrm{S}_{\mathrm{MHD}}}{J}\right)_{i}^{k+1}
\end{aligned}
$$

and the superscript $k+1$ denotes the current iteration (i.e. sweep) level. In the preceding equation, the MHD source term, $\mathbf{S}_{\mathbf{M H D}}$, is treated explicitly since it is evaluated using the velocity at station $\mathrm{i}\left(\mathbf{V}_{\boldsymbol{i}}\right)$. For most cases, this will not degrade the accuracy of the solution since $\Delta \xi$ is small and the velocity changes slowly. If this is not the case, a predictor-corrector procedure can be implemented whereby a predicted velocity at station $i+1\left(V_{\widetilde{i+1}}\right)$ is first obtained using Eq. (24). The solution at station $i+1$ is then recomputed by evaluating $S_{M H D}$ with $\mathbf{V}_{i+1}$.

\section{Numerical Results}

The numerical calculation of the supersonic flow in the experimental MHD channel is now discussed. This flowfield was previously computed by MacCormack [12] using the full N-S equations coupled with the electromagnetodynamic equations. The electrical conductivity in his calculations was set at $1.0 \times 10^{5}$ $\mathrm{mho} / \mathrm{m}$ resulting in a very large magnetic Reynolds number. In the present study, the calculations are performed in the low magnetic Reynolds number regime using a realistic value of electrical conductivity. Both 2-D and 3-D results have been obtained.

The flow in the nozzle section was computed using a combination of the OVERFLOW code [28] and the present PNS code (without MHD effects). For the 3D OVERFLOW nozzle calculation, a highly stretched grid consisting of $130 \times 50 \times 50$ grid points was used. The normal grid spacing at the wall was $1.0 \times 10^{-5}$ $m$. For the PNS calculation of the flow in the remainder of the nozzle and the rest of the MHD channel, a highly stretched grid consisting of 60 points in both the $y$ and $z$ directions was used and the normal grid spacing at the wall was $1.0 \times 10^{-5} \mathrm{~m}$. As 
a consequence of flow symmetry, only one-fourth of the channel cross section was computed in the 3-D calculations.

The calculations were performed assuming turbulent flow throughout the MHD channel. In addition the flow was computed using a constant $\bar{\gamma}$ of 1.25 to simulate equilibrium air. The channel wall temperature was assumed to be isothermal since steady flow conditions were maintained in the experiment for only about 1.2 milliseconds. A schematic of the powered portion of the MHD channel with the directions of the applied magnetic and electric fields is shown in Fig. 2. The values of the electrical conductivity $\left(\sigma_{e}\right)$, the magnetic field $\left(B_{z}\right)$, and the electric field $\left(E_{y}\right)$ were kept constant in the powered portion of the channel. Two test cases corresponding to Runs 15 and 16 of the NASA Ames experiments [29] were computed in this study and are now discussed.

\section{Test Case 1: NASA Ames MHD Run 15 $\left(V_{\text {cap. }}=320 \mathrm{~V}\right)$}

The dimensional flow parameters for this test case are:

$$
\begin{aligned}
p_{o} & =9.10 \times 10^{5} \mathrm{~N} / \mathrm{m}^{2} \\
T_{0} & =5560 \mathrm{~K} \\
T_{w} & =300 \mathrm{~K} \\
\sigma_{e} & =130 \mathrm{mho} / \mathrm{m} \\
B_{z} & =0.0,0.92 \mathrm{~T} \\
E_{y} & =0,3955,5000,6000 \mathrm{~V} / \mathrm{m}
\end{aligned}
$$

where the subscript o denotes total conditions at the nozzle entrance and $w$ denotes wall conditions.

This case was computed using several different electric field strengths in order to properly simulate the experiment. In the experiment, the voltage applied to the electrodes was approximately $134 \mathrm{~V}$ for this case, however, due to the sheath voltage drop, the actual voltage applied to the flow is smaller than the electrode voltage. The voltage drop was measured for the central inviscid core flow, and was approximately $67 \mathrm{~V}$ [2]. Since the boundary layer is computed in the numerical solution, the applied electric field must be approximately the voltage drop across the electrodes minus the sheath voltage drop. Unfortunately, it is not a trivial task to measure the sheath voltage drop. Therefore, several different electric fields were chosen in the numerical calculations so that the corresponding voltage drop across the electrodes would be between $67 \mathrm{~V}$ and $134 \mathrm{~V}$. The voltage drop of $67 \mathrm{~V}$ corresponds to $E_{y}=3955 \mathrm{~V} / \mathrm{m}$ at the center of the accelerator section and a voltage drop of $101.6 \mathrm{~V}$ corresponds to $E_{y}=6000 \mathrm{~V} / \mathrm{m}$, with a sheath voltage drop of $32.4 \mathrm{~V}$.
The computed streamwise variation of static pressure for the 2-D calculations is shown in Fig. 3 for the different electric field strengths. The pressure variation with no electric field or magnetic field is denoted by $E_{y}=0$. The results are in reasonable agreement with the experiment. The numerical results show an increase in static pressure as the electric field strength is increased.

The computed streamwise variation of averaged velocity for the 2-D calculations is shown in Fig.:4. The velocities are averaged across the channel cross section and normalized using the entrance velocity to be consistent with the experiment. In the experiment, the velocities were obtained by measuring the voltage generated by the flow at the last electrode pair (19) which is unpowered. This procedure inherently involves an averaging of the velocity profile. The numerical results indicate an increase in the averaged velocity of about $26 \%$ with $E_{y}=6000 \mathrm{~V} / \mathrm{m}$ and this compares well with the experimental value of approximately $27 \%$. The velocity vector plots for $E_{y}=0$ and $E_{y}=6000 \mathrm{~V} / \mathrm{m}$ are shown in Fig. 5.

The computed streamwise variation of averaged velocity for the 3-D calculations is shown in Fig. 6. The velocities are averaged and normalized in the same manner as for the 2-D calculations. The numerical results indicate an increase in the averaged velocity of about $26 \%$ with $E_{y}=6000 \mathrm{~V} / \mathrm{m}$ which is the same value obtained with the $2-D$ calculations.

\section{Test Case 2: NASA Ames MHD Run 16 $\left(V_{\text {cap. }}=380 \mathrm{~V}\right)$}

The dimensional flow parameters for this test case are:

$$
\begin{aligned}
p_{o} & =9.92 \times 10^{5} \mathrm{~N} / \mathrm{m}^{2} \\
T_{o} & =5560 \mathrm{~K} \\
T_{w} & =300 \mathrm{~K} \\
\sigma_{e} & =140 \mathrm{mho} / \mathrm{m} \\
B_{z} & =0.0,0.92 \mathrm{~T} \\
E_{y} & =0,4309,5000,6000,7000 \mathrm{~V} / \mathrm{m}
\end{aligned}
$$

This test case was also computed using several different electric field strengths in order to properly simulate the experiment. The computed streamwise variation of static pressure for the 2-D calculations is shown in Fig. 7 for the different electric field strengths. The computed pressures are in good agreement with the experimental pressures. The computed streamwise variation of averaged velocity for the $2-D$ calculations is shown in Fig. 8. The numerical results indicate an increase in the averaged velocity of about $31 \%$ with $E_{y}=7000 \mathrm{~V} / \mathrm{m}$. This is less than the value of $38 \%$ that was obtained in the experiment. The corresponding streamwise variation of averaged velocity 
for the 3-D calculations is shown in Fig. 9. The numerical results indicate an increase in the averaged velocity of about $30 \%$ with $E_{y}=7000 \mathrm{~V} / \mathrm{m}$ which is again less than the experimental value of $38 \%$.

\section{Concluding Remarks}

In this study, a new 3-D parabolized Navier-Stokes algorithm has been developed to efficiently compute MHD flows in the low magnetic Reynolds number regime. The new algorithm has been used to compute the flow in the NASA Ames experimental-MHD channel for Runs 15 and 16. The numerical results are in good agreement with most of the experimental results.

\section{Acknowledgments}

This work was supported by NASA Ames Research Center under Grant NCC2-5517 and by Iowa State University. The Technical Monitor for this grant is Dr. Unmeel B. Mehta. The authors wish to thank Dr. David W. Bogdanoff of NASA Ames for his help and comments regarding the MHD channel experiments.

\section{References}

[1] Bogdanoff, D. W., Park, C., and Mehta, U. B., "Experimental Demonstration of MagnetoHydrodynamic (MHD) Acceleration - Facility and Conductivity Measurements," NASA TM2001-210922, July 2001.

[2] Bogdanoff, D. W. and Mehta, U. B., "Experimental Demonstration of Magneto-HydroDynamic (MHD) Acceleration," AIAA Paper 2003-4285, June 2003.

[3] Gaitonde, D. V., "Development of a Solver for 3D Non-Ideal Magnetogasdynamics," AIAA Paper 99-3610, June 1999.

[4] Damevin, H. M., Dietiker, J.-F., and Hoffmann, K. A., "Hypersonic Flow Computations with Magnetic Field," AIAA Paper 2000-0451, Jan. 2000.

[5] Hoffmann, K. A., Damevin, H. M., and Dietiker, J.-F., "Numerical Simulation of Hypersonic Magnetohydrodynamic Flows," AIAA Paper 2000-2259, June 2000.
[6] Gaitonde, D. V. and Poggie, J., "Simulation of MHD Flow Control Techniques," AIAA Paper 2000-2326, June 2000.

[7] MacCormack, R. W., "Numerical Computation in Magnetofluid Dynamics," Computational Fluid Dynamics for the 21st Century, Kyoto, Japan, July 2000.

[8] Deb, P. and Agarwal, R., "Numerical Study of MHD-Bypass Scramjet Inlets with Finite-Rate. Chemistry," AIAA Paper 2001-0794, Jan. 2001.

[9] MacCormack, R. W., "A Computational Method for Magneto-Fluid Dynamics," AIAA Paper 2001-2735, June 2001.

[10] Gaitonde, D. V. and Poggie, J., "An Implicit Technique for 3-D Turbulent MGD with the Generalized Ohm's Law," AIAA Paper 20012736, June 2001.

[11] Dietiker, J.-F. and Hoffmann, K. A., “Numerical Simulation of Turbulent Magnetohydrodynamic Flows," AIAA Paper 2001-2737, June 2001.

[12] MacCormack, R. W., "Three Dimensional Magneto-Fluid Dynamics Algorithm Development," AIAA Paper 2002-0197, Jan. 2002.

[13] Munipalli, R, Anderson, D. A., and Kim, H., "Two-Temperature Computations of Ionizing Air with MHD Effects," AIAA Paper 2000-0450, Jan. 2000.

[14] Dietiker, J.-F. and Hoffmann, K. A., "Boundary Layer Control in Magnetohydrodynamic Flows," AIAA Paper 2002-0130, Jan. 2002.

[15] Cheng, F., Zhong, X., Gogineni, S., and Kimmel, R. L., "Effect of Applied Magnetic Field on the Instability of Mach 4.5 Boundary Layer over a Flat Plate," AIAA Paper 2002-0351, Jan. 2002.

[16] Tannehill, J. C., Anderson, D. A., and Pletcher, R. H., Computational Flusid Mechanics and Heat Transfer, Taylor and Francis, Washington, D.C., 1997.

[17] Kato, H., Tannehill, J. C., Ramesh, M. D., and Mehta, U. B., "Computation of Magnetohydrodynamic Flows Using an Iterative PNS Algorithm," AIAA Paper 2002-0202, Jan. 2002.

[18] Kato, H., Tannehill, J. C., and Mehta, U. B., "Numerical Simulation of Turbulent MHD Flows Using an Iterative PNS Algorithm," AIAA Paper 2003-0326, Jan. 2003. 
[19] Lawrence, S. L., Tannehill, J. C., and Chaussee, D. S., "Upwind Algorithm for the Parabolized Navier-Stokes Equations," AIAA Journal, Vol. 27, No. 9, Sept. 1989, pp. 1975-1983.

[20] Miller, J. H., Tannehill, J. C., and Lawrence, S. L., "PNS Algorithm for Solving Supersonic Flows with Upstream Influences," AIAA Paper 98-0226, Jan. 1998.

[21] Tannehill, J. C., Miller, J. H., and Lawrence; S. L., "Development of an Iterative PNS Code for Separated Flows," ALAA Paper 99-3361, June 1999.

[22] Kato, H. and Tannehill, J. C., "Development of a Forward-Backward Sweeping Parabolized Navier-Stokes Algorithm," AIAA Paper 20020735, Jan. 2002.

[23] Srinivasan, S., Tannehill, J. C., and Weilmuenster, K. J., "Simplified Curve Fits for the Thermodynamic Properties of Equilibrium Air," NASA RP 1181, Aug. 1987.

[24] Srinivasan, S., Tannehill, J. C., and Weilmuenster, K. J., "Simplified Curve Fits for the Transport Properties of Equilibrium Air," NASA CR $178411,1987$.

[25] Baldwin, B. S. and Lomax, H., "Thin Layer Approximation and Algebraic Model for Separated Turbulent Flows," AIAA-Paper 78-257, Jan. 1978.

[26] Lykoudis, P. S., "Magneto Fluid Mechanics Channel Flow, II Theory," The Physics of Fluids, Vol. 10, No. 5, May 1967, pp. 1002-1007.

[27] Vigneron, Y. C., Rakich, J. V., and Tannehill, J. C., "Calculation of Supersonic Flow over Delta Wings with Sharp Subsonic Leading Edges," ALAA Paper 78-1137, July 1978.

[28] Buning, P. G., Jespersen, D. C., and Pulliam, T. H., OVERFLOW Manual, Version 1.7v, NASA Ames Research Center, Moffett Field, California, June 1997.

[29] Bogdanoff, D. W., Private Communication, Dec. 1,2003 


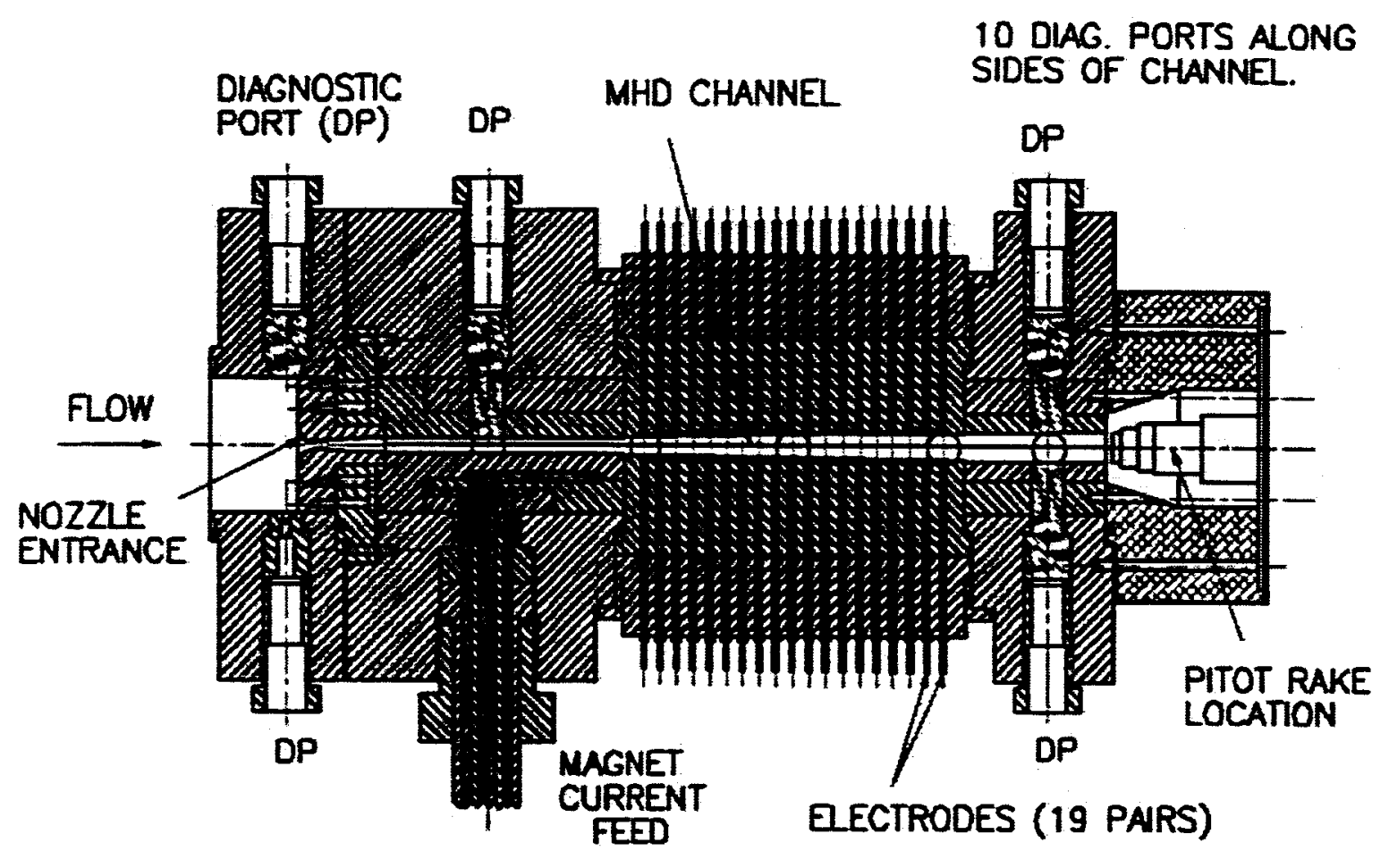

Figure 1: Cross section of NASA Ames MHD Channel

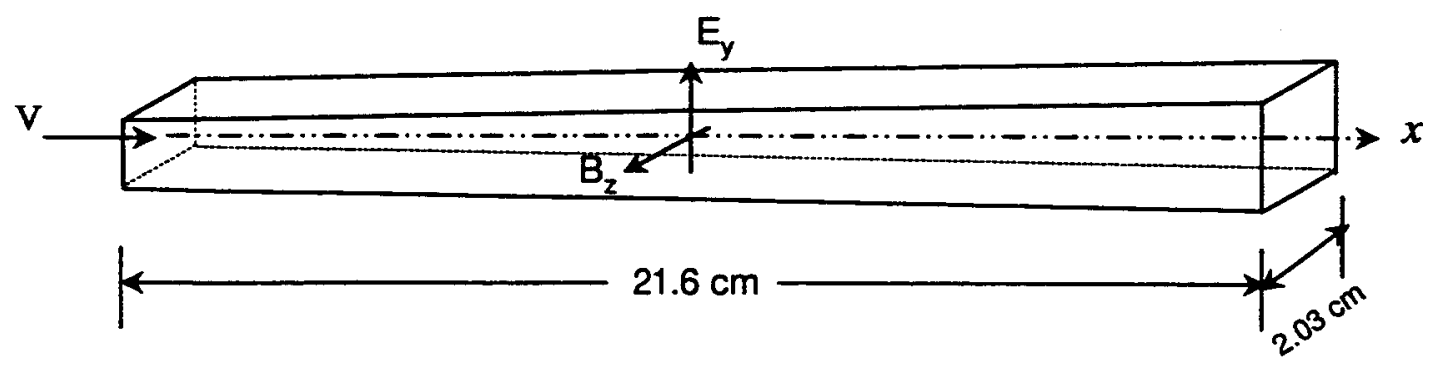

Figure 2: Schematic of powered portion of MHD channel 


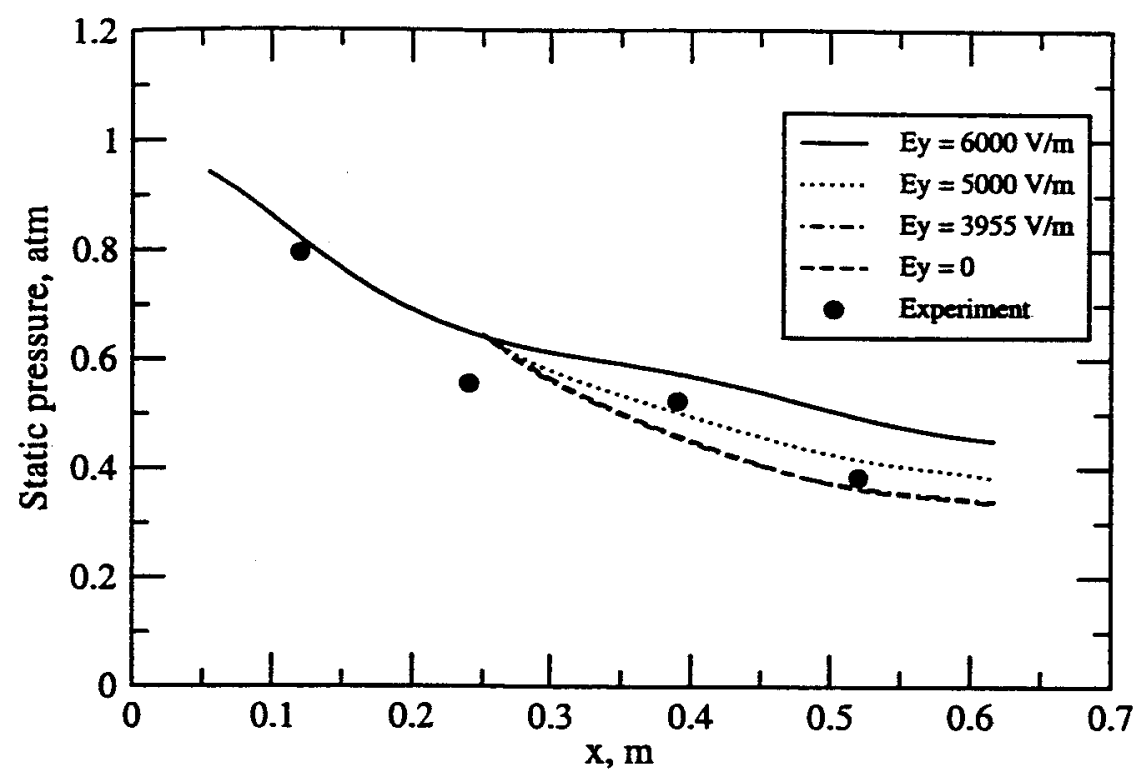

Figure 3: Streamwise variation of static pressure (2-D)

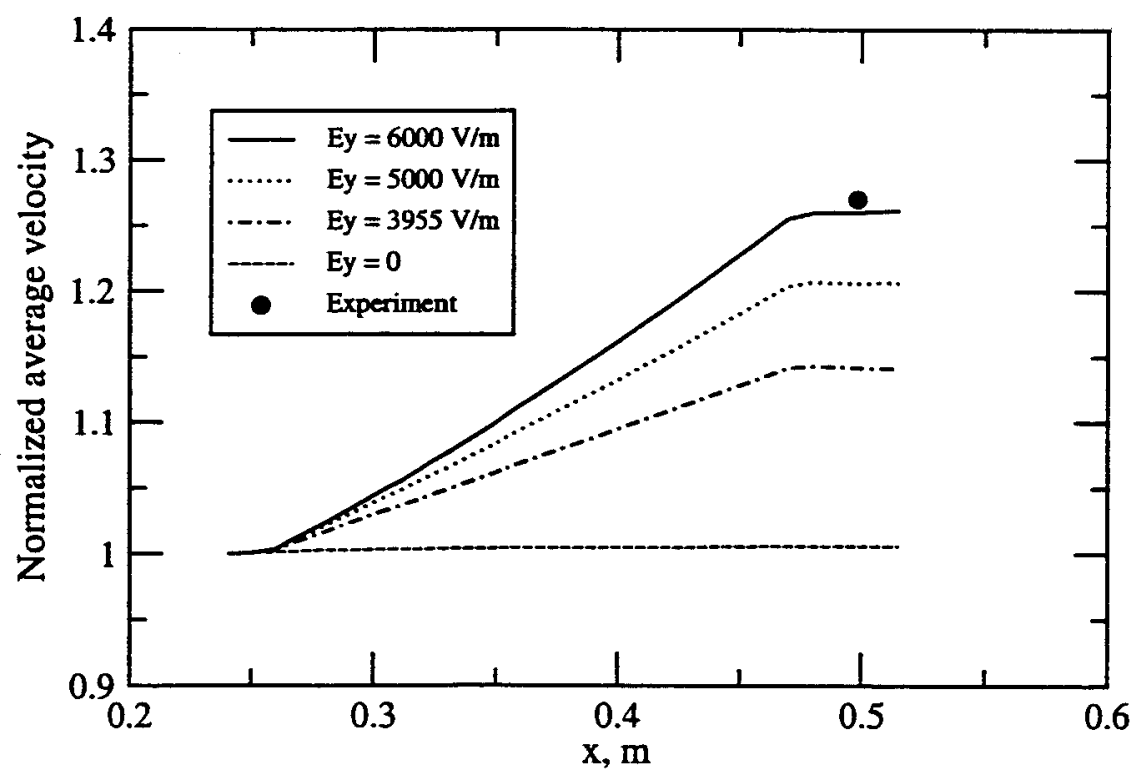

Figure 4: Streamwise variation of averaged velocity (2-D) 


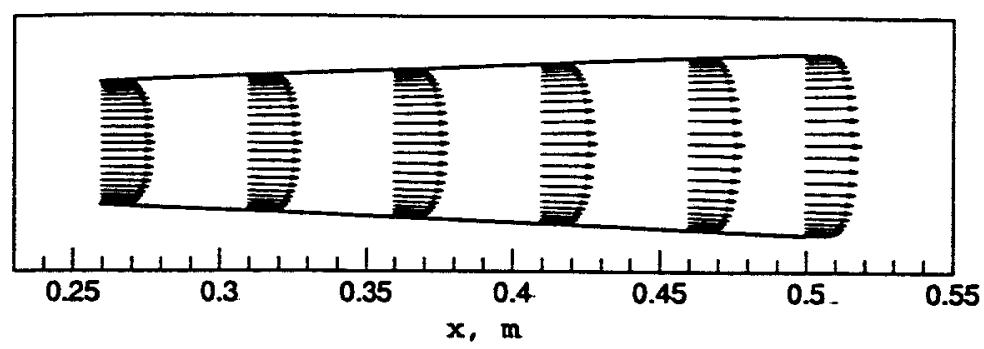

(a) $E_{\boldsymbol{y}}=0$

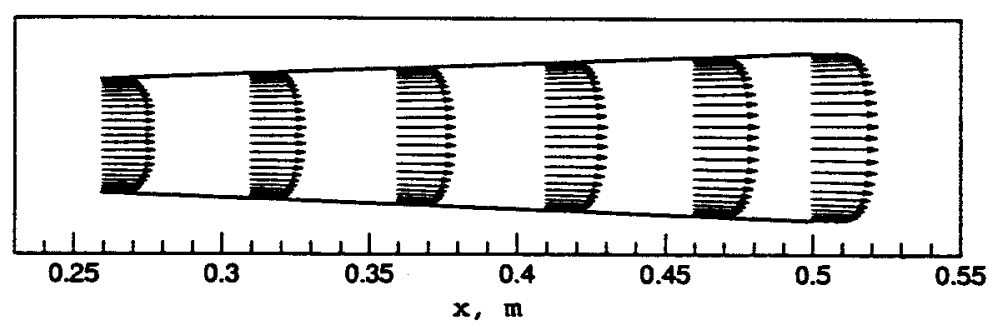

(b) $E_{y}=6000 \mathrm{~V} / \mathrm{m}$

Figure 5: Velocity vectors along streamwise direction (2-D)

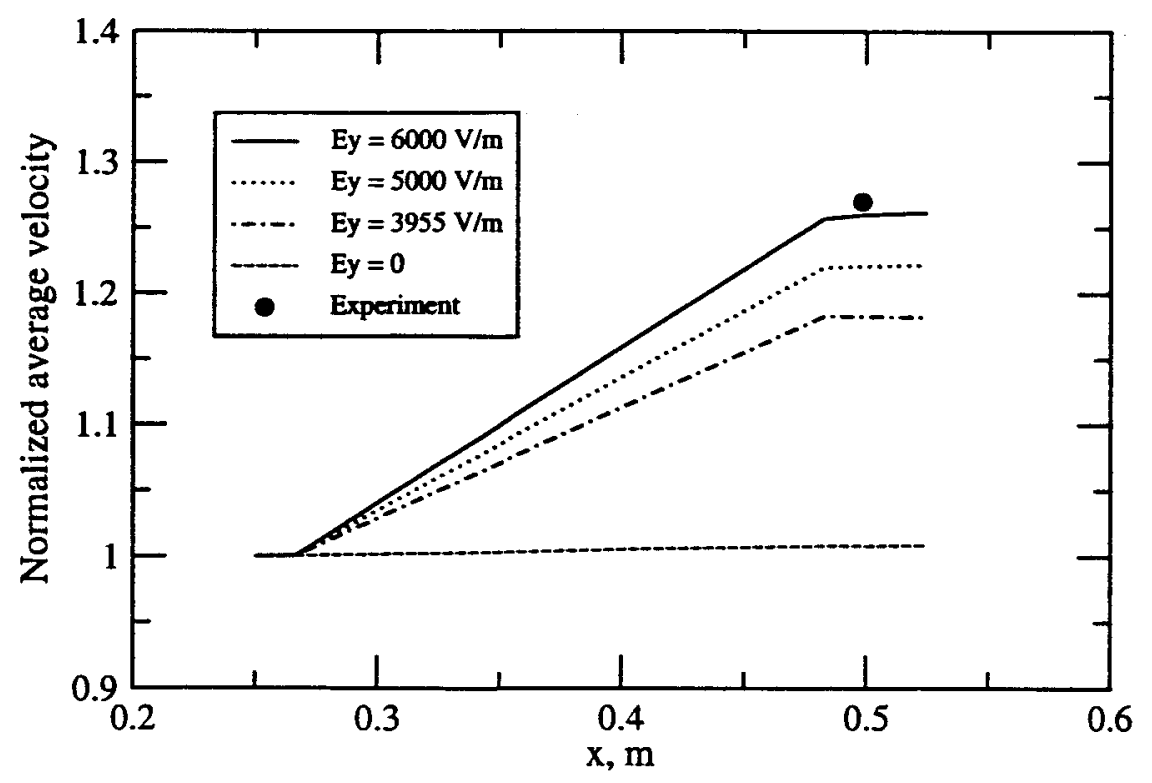

Figure 6: Streamwise variation of averaged velocity (3-D) 


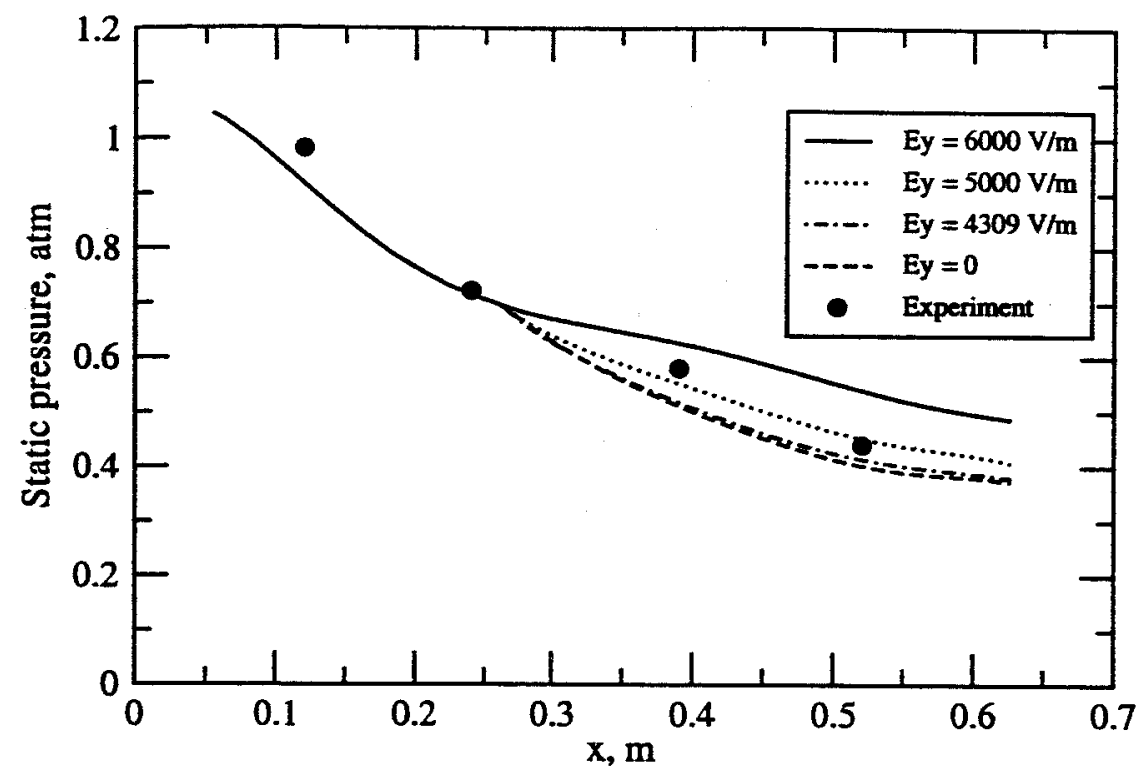

Figure 7: Streamwise variation of static pressure (2-D)

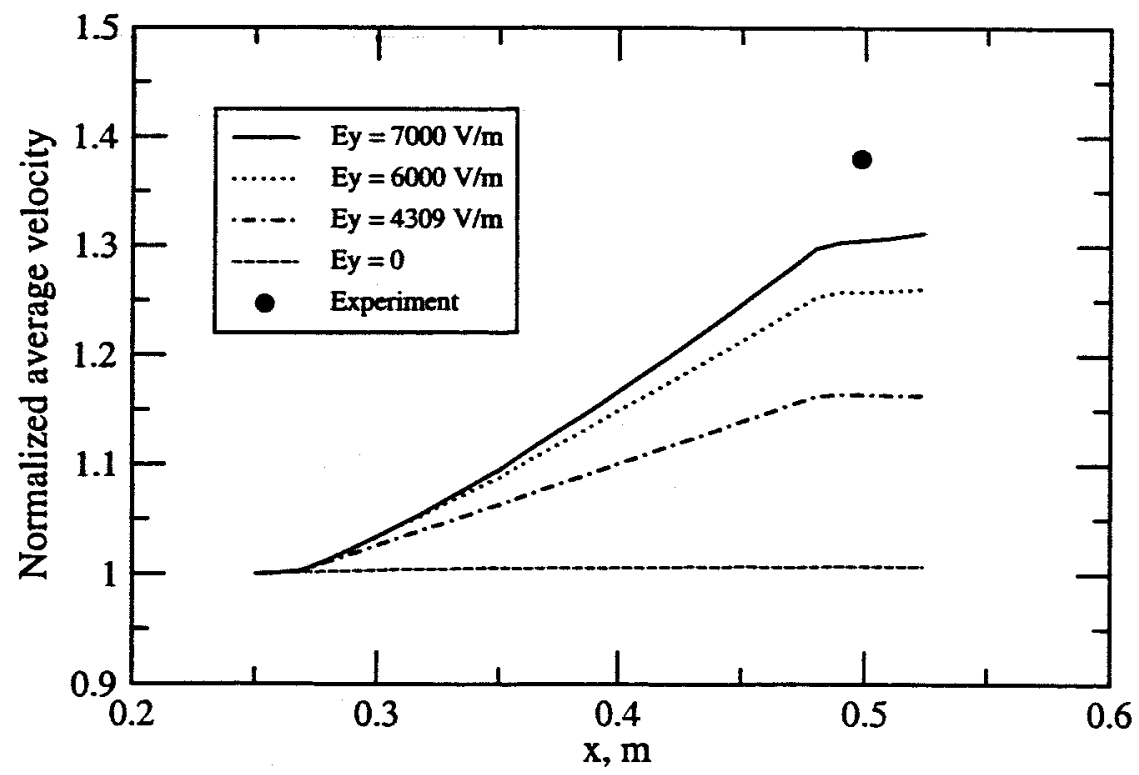

Figure 8: Streamwise variation of averaged velocity (2-D) 


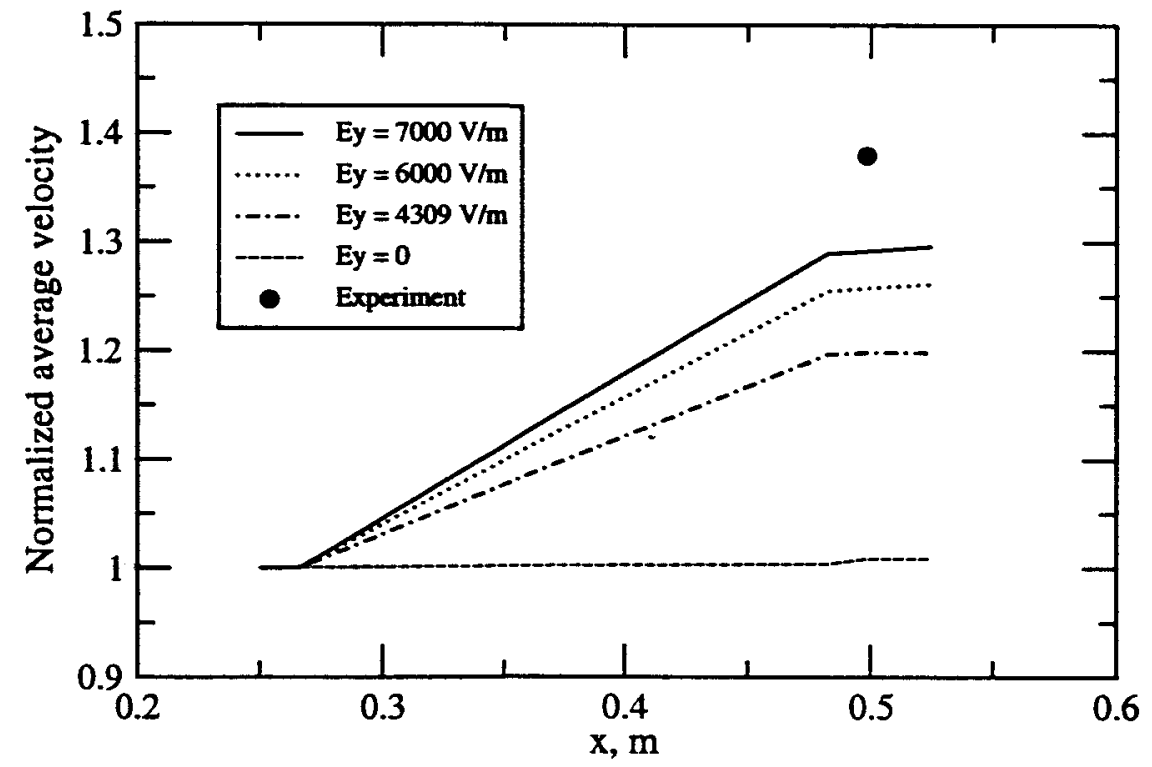

Figure 9: Streamwise variation of averaged velocity (3-D) 